\title{
Bivariate extension of the Pickands-Balkema-de Haan theorem
}

\author{
Mario V. Wüthrich \\ Winterthur Insurance, Römerstrasse 17, P.O. Box 357, CH-8401 Winterthur, Switzerland
}

Received 16 September 2002; accepted 25 March 2003

\begin{abstract}
We prove a two-dimensional version of the famous Pickands-Balkema-de Haan theorem of extreme value theory. The bivariate random variables are generated using the copula language. This representation of dependence structures allows to derive asymptotic results for bivariate excess distributions.

(c) 2003 Elsevier SAS. All rights reserved.

\section{Résumé}

Une version en dimension 2 du célèbre théorème de Pickands-Balkema-de Haan sur la théorie des valeurs extrêmes est démontrée. Les variables aléatoires bivariées sont générées en utilisant le langage des copules. Cette représentation des structures de dépendance permet de dériver des résultats asymptotiques pour les distributions d'excès bivariées.

(c) 2003 Elsevier SAS. All rights reserved.
\end{abstract}

MSC: 62E20; 62H20; 62P05

Keywords: Archimedean copula; Dependent random variables; Extreme value theory; Pickands-Balkema-de Haan theorem

\section{Introduction}

Copulas were originally introduced about 40 years ago in the context of probabilistic metric spaces. During the past years they have developped rapidly and they have attracted much interest (see, e.g., Kotz-Nadarajah [10]). Copulas are used to describe scale invariant dependencies between random variables. An understanding of such stochastic dependence structures has become very important in all fields of probability theory. Especially in the actuarial world, copulas have proven their usefulness for constructing appropriate multivariate models. An introduction and overviews over recent developments and applications can be found in Joe [7], Nelsen [11], Frees and Valdez [4], Wüthrich [16], Embrechts, McNeil and Straumann [3] and the references therein.

In general it is quite difficult to fit an explicit model to real data. Therefore one is interested into asymptotic behaviours and theorems in order to approximate the true problem by its asymptotic results (central limit theorem, large deviations results, etc.). In this article we prove a two-dimensional generalizations of the Pickands-Balkema-

E-mail address: mario.wuethrich@ winterthur.ch (M.V. Wüthrich). 
de Haan theorem (see Pickands [12] and Balkema and de Haan [1]) stating that the generalized Pareto distribution given by

$$
G_{\zeta, \beta}(x)= \begin{cases}1-(1+\zeta x / \beta)^{-1 / \zeta}, & \zeta \neq 0 \\ 1-\exp (-x / \beta), & \zeta=0\end{cases}
$$

where $\beta>0$, and $x \geqslant 0$ for $\zeta \geqslant 0$ or $0 \leqslant x \leqslant-\beta / \zeta$ for $\zeta<0$, appears as the limit distribution of scaled excesses over high thresholds. More precisely, it can be shown (see [2], Theorem 3.4.13(b)) that for a large class of random variables $X$ there exists a function $\beta(\cdot)$ such that

$$
\lim _{u \rightarrow x_{0}} \sup _{0 \leqslant x<x_{0}-u}\left|P[X-u \leqslant x \mid X>u]-G_{\zeta, \beta(u)}(x)\right|=0,
$$

where $x_{0} \leqslant \infty$ denotes the right endpoint of the distribution function of $X$.

Organization of this paper. In the next section we give all definitions and our results (Theorem 2.5 and Lemma 2.6). The idea is to define bivariate random variables via the copula construction, which "separates" the marginal distributions from the dependence structure (see Theorem 2.2). This decomposition allows to separately analyze asymptotic behaviours of marginals and the dependence structure, respectively. This construction leads to similar results as (1.2) in a bivariate context (see Theorem 2.5 below). The limiting distributions (which play the role of the generalized Pareto distribution in (1.2)) are analyzed in Lemma 2.6.

In Section 3 we give examples. The best known examples are the so-called Archimedean copulas which are considered in Section 3.1 and the Gaussian copula which is treated in Section 3.2. One main observation is that Gaussian models usually underestimate joint extreme values in practice, since bivariate Gaussian random variables are asymptotically independent (see also [3]).

Finally in Section 4 we give the proofs of all our results.

\section{Definitions and results}

\subsection{Multivariate copulas}

To generate bivariate random variables we use the copula approach. The idea behind the concept of copulas is to separate a multivariate distribution function into two parts, one describing the dependence structure and the other one describing marginal behaviours, respectively.

Definition 2.1 (Copula). Choose $d \geqslant 2$. A $d$-dimensional copula is a $d$-dimensional distribution function restricted to $[0,1]^{d}$ with uniform- $(0,1)$ marginals.

Theorem 2.2 (Sklar $[15,13,11])$. For a given joint distribution function $F$ with continuous marginals $F_{1}, \ldots, F_{d}$ there exists a unique copula $C$ satisfying

$$
F\left(x_{1}, \ldots, x_{d}\right)=C\left(F_{1}\left(x_{1}\right), \ldots, F_{d}\left(x_{d}\right)\right) .
$$

Conversely, for a given copula $C$ and marginals $F_{1}, \ldots, F_{d}$ we have that (2.1) defines a distribution with marginals $F_{i}$.

Sklar's threorem is a motivation for calling a copula a dependence structure. In fact, (2.1) means that $C$ couples the marginals $F_{i}$ to the joint distribution function $F$. One important property of copulas is that they are invariant under monotonically increasing transformations (i.e. they do not depend on marginals).

There are several special copulas, e.g., the so called comonotonic copula which corresponds to total positive dependence or the independent copula which is the copula of independent random variables:

$$
C_{\mathrm{I}}\left(x_{1}, \ldots, x_{d}\right)=x_{1} \cdots x_{d}
$$

for more background information we refer to [3] (more examples are given below). 


\subsection{Marginal behaviour}

In the Fisher-Tippett theorem and the Pickands-Balkema-de Haan theorem (see, e.g., Theorems 3.2.3 and 3.4.13(b) in [2]) one sees that the one-dimensional distributions (marginals) can essentially be divided into three subclasses for analyzing extremal events. The crucial conditition is the rate of decay at $\pm \infty$, i.e. the fatness of the tails:

We introduce some notations: A function $h$ is regularly varying at $-\infty$ with index $\alpha$, write $h \in \mathcal{R}_{\alpha}$, if

$$
\lim _{x \rightarrow-\infty} \frac{h(x t)}{h(x)}=t^{\alpha}, \quad t>0 .
$$

Let $x_{F}$ denote the left-endpoint of the distribution $F$. The three subclasses are given by

- Fréchet case: $F(\cdot) \in \mathcal{R}_{-\beta}$ for $\beta>0$.

- Weibull case: Assume $x_{F}>-\infty$ and $F\left(x_{F}-1 / \cdot\right) \in \mathcal{R}_{-\beta}$ for $\beta>0$.

- Gumbel case: Assume $x_{F} \geqslant-\infty$ and there exists a positive function $a(\cdot)$ such that for $t \in \mathbb{R}$

$$
\lim _{u \downarrow x_{F}} \frac{F(u+t a(u))}{F(u)}=\mathrm{e}^{t} .
$$

For examples we refer to [2], Chapter 3. Some examples are given below.

\subsection{Main results: bivariate excess distributions below low thresholds}

In this subsection we state our main theorem. It gives a weak convergence result for the distribution of peaks below low thresholds. We remark that from a technical point of view it is easier to prove results for peaks below low thresholds than results for peaks over high thresholds. This comes from the fact that excess copulas are not symmetric for lower and upper tails. The upper tails are treated in a sequel of this work (see Juri and Wüthrich [9]).

Assumption 2.3. Assume $(X, Y)$ is a symmetric random vector with copula $C(\cdot, \cdot)$ and continuous marginals $F$, i.e. $(X, Y) \stackrel{(d)}{=}(Y, X)$ and

$$
P[X \leqslant x, Y \leqslant y]=C(F(x), F(y)) .
$$

Furthermore we assume that there exists a continuous function $g: \mathbb{R}_{+} \rightarrow \mathbb{R}_{+}$with $g(x)>0$ for all $x>0$ and the following limit exists with

$$
\lim _{u \rightarrow 0} \frac{C(x u, u)}{C(u, u)}=g(x), \quad \text { for all } x \in \mathbb{R}_{+} .
$$

Remarks. Assumption 2.3 is quite natural if we think that we have exchangeable random variables with dependence structure $C$. If we take $C(x, y)=C_{I}(x, y)=x \cdot y$, which corresponds to independent random variables, then $g(x)=x$. We see below that the function $g$ characterizes the asymptotic behaviour of the dependence structure for extremal events.

Lemma 2.4. Assume $(X, Y)$ satisfies Assumption 2.3. Then $g$ is an increasing function with $g(0)=0$ and $g(1)=1$. Furthermore there exists $\theta \in \mathbb{R}$ such that

$$
g(x)=x^{\theta} g\left(\frac{1}{x}\right) \text { for all } x>0 .
$$


Now we are able to formulate our main theorem which is a bivariate version of the Pickands-Balkema-de Haan theorem (see [12,1]). For $(X, Y)$ satisfying Assumption 2.3 we find $\theta \in \mathbb{R}$ such that Lemma 2.4 is satisfied. Then we define for $x, y \geqslant 0$

$$
G(x, y)= \begin{cases}y^{\theta} g(x / y) & x>0 \text { and } y>0 \\ 0 & x=0 \text { or } y=0\end{cases}
$$

Theorem 2.5 (Excesses below low thresholds). Assume $(X, Y)$ satisfies Assumption 2.3. Choose $0<x_{1} \leqslant x_{2}$ and $0<y_{1} \leqslant y_{2}$. Then we have:

(a) Fréchet case: marginals $F(\cdot) \in \mathcal{R}_{-\beta}, \beta>0$,

$$
\lim _{u \rightarrow-\infty} P\left[X \leqslant \frac{u}{x_{1}}, Y \leqslant \frac{u}{y_{1}} \mid X \leqslant \frac{u}{x_{2}}, Y \leqslant \frac{u}{y_{2}}\right]=\frac{G\left(x_{1}^{\beta}, y_{1}^{\beta}\right)}{G\left(x_{2}^{\beta}, y_{2}^{\beta}\right)} .
$$

(b) Weibull case: marginals $F$ with $x_{F}>-\infty$ and $F\left(x_{F}-1 / \cdot\right) \in \mathcal{R}_{-\beta}, \beta>0$,

$$
\lim _{u \rightarrow-\infty} P\left[X \leqslant x_{F}-\frac{x_{1}}{u}, Y \leqslant x_{F}-\frac{y_{1}}{u} \mid X \leqslant x_{F}-\frac{x_{2}}{u}, Y \leqslant x_{F}-\frac{y_{2}}{u}\right]=\frac{G\left(x_{1}^{\beta}, y_{1}^{\beta}\right)}{G\left(x_{2}^{\beta}, y_{2}^{\beta}\right)} .
$$

(c) Gumbel case: marginals $F$ with $x_{F} \geqslant-\infty$ and there exists a positive function a (.) such that (2.4) is satisfied, we have

$$
\lim _{u \rightarrow-\infty} P\left[X \leqslant u+x_{1} a(u), Y \leqslant u+y_{1} a(u) \mid X \leqslant u+x_{2} a(u), Y \leqslant u+y_{2} a(u)\right]=\frac{G\left(\mathrm{e}^{x_{1}}, \mathrm{e}^{y_{1}}\right)}{G\left(\mathrm{e}^{x_{2}}, \mathrm{e}^{y_{2}}\right)} .
$$

Remark. Of course we have similar results for excess distributions over high thresholds. However the formulas become more complicated for peaks-over-thresholds since copulas are not symmetric under "sign flip"-operations $X \mapsto-X$ (not all the signs point into the same direction). Therefore we omit the formulas for excess distributions over high thresholds, rigorous results can be found in Juri and Wüthrich [9], Theorem 4.1.

For the limiting functions $G$ we have the following lemma:

Lemma 2.6. $G(\cdot, \cdot)$ is a positive, continuous, symmetric function which is increasing in both arguments. Furthermore it satisfies the two-increasing property. Hence $G(\cdot, \cdot) / G\left(x_{2}, y_{2}\right)$ defines a two-dimensional probability distribution on $\left[0, x_{2}\right] \times\left[0, y_{2}\right]$.

Remark. In Theorem 2.5 we have seen that the function $G$ plays the role of an attractor. Therefore one would believe that $G$ satisfies certain invariance properties. Indeed this is the case: if we consider the copula generated by $G$, then one sees that this copula is invariant under "lower tail dependence copula"-transformations (for detailed results see Juri and Wüthrich [9], Theorem 2.9).

\subsection{Interpretation and conclusions}

Theorem 2.5 says that bivariate excess distributions can asymptotically be described by $G$ (we have a weak convergence result for excess distributions). $G$ can solely be determined by the asymptotic behaviour of the dependence structure (definition of $g$ ) and the asymptotic behaviour of the marginals. In the examples below we see that the asymptotic behaviour of the copula can often be described by one parameter which plays the role of the dependence strength, i.e. in these cases the limit distribution can be parameterized by two or three parameters.

For practical purposes this is quite an important result: Often one has not enough data to analyze the behaviour of extremal events, hence we approximate the true problem by its asymptotic bound (e.g., Pickands-Balkema-de Haan theorem). I.e. we have to estimate two or three parameters from the data to determine the limiting distribution. This limiting distribution is then used to estimate the "true" problem. The remaining questions then are algorithms to estimate the parameters and the question about speed of convergences. These are not studied in this article. 


\section{Examples}

\subsection{Archimedean copulas}

There is an easy way to construct copulas, see also [4-6,11]. The so-called Archimedean copulas are generated as follows:

Definition 3.1 (Archimedean copulas). Let $\psi:[0,1] \rightarrow[0, \infty]$ be strictly decreasing, convex and such that $\psi(0)=\infty$ and $\psi(1)=0$. Define for $x_{i} \in[0,1], i=1,2$,

$$
C^{\psi}\left(x_{1}, x_{2}\right)=\psi^{-1}\left(\psi\left(x_{1}\right)+\psi\left(x_{2}\right)\right) \text {. }
$$

The function $\psi$ is called generator of $C^{\psi}$.

Remark. $C^{\psi}$ is a two-dimensional copula (see [5,6,11]). Archimedean copulas are interesting in practice because they are very easy to construct, but still we obtain a rich family of dependence structures. Usually they have only one parameter which is a great advantage when one needs to estimate parameters from data. We give two examples, for more examples we refer, e.g., to [8].

\section{Examples of Archimedean copulas}

- The Clayton copula with $\alpha>0$ is generated by $\psi(t)=t^{-\alpha}-1$ and takes the form

$$
C^{\mathrm{Cl}, \alpha}\left(x_{1}, x_{2}\right)=\left(x_{1}^{-\alpha}+x_{2}^{-\alpha}-1\right)^{-1 / \alpha} .
$$

- The Gumbel copula with $\alpha \geqslant 1$ is generated by $\psi(t)=(-\log (t))^{\alpha}$ and takes the form

$$
C^{\mathrm{Gu}, \alpha}\left(x_{1}, x_{2}\right)=\exp \left[-\left\{\left(-\log x_{1}\right)^{\alpha}+\left(-\log x_{2}\right)^{\alpha}\right\}^{1 / \alpha}\right] .
$$

For our two dependence structures we calculate the limit (2.6), which gives $g(\cdot)$.

Example 3.2 (Clayton copula). We choose $x>0$, then

$$
\lim _{v \rightarrow 0} \frac{C^{\mathrm{Cl}, \alpha}(x v, v)}{C^{\mathrm{Cl}, \alpha}(v, v)}=\lim _{v \rightarrow 0}\left(\frac{(x v)^{-\alpha}+v^{-\alpha}-1}{2 v^{-\alpha}-1}\right)^{-1 / \alpha}=\left(\frac{x^{-\alpha}+1}{2}\right)^{-1 / \alpha}=g(x) .
$$

An easy calculation shows that $\theta=1$ in this example (see Lemma 2.4). Hence we calculate

$$
G^{\mathrm{Cl}, \alpha}(x, y)=y \cdot g\left(\frac{x}{y}\right)=\left(\frac{x^{-\alpha}+y^{-\alpha}}{2}\right)^{-1 / \alpha} \text {. }
$$

Hence

$$
G^{\mathrm{Cl}, \alpha}(\cdot, \cdot) / G^{\mathrm{Cl}, \alpha}\left(x_{2}, y_{2}\right)
$$

defines a two-dimensional distribution on $\left[0, x_{2}\right] \times\left[0, y_{2}\right]$. The remarkable thing is that this distribution has again Clayton copula (one can easily see this using Sklar's theorem). In view of [8], Theorem 3.3, this result is not surprising because the Clayton copula is invariant under excess distribution transformations.

We choose marginals which belong to the Fréchet case. Assume $F$ is a Pareto distribution with parameters $\theta, \beta>0$, i.e. for $x \leqslant-\theta$ we have

$$
F(x)=(\theta /-x)^{\beta} \in \mathcal{R}_{\beta} .
$$

Hence for $(X, Y) \stackrel{(d)}{\sim} C^{\mathrm{Cl}, \alpha}(F(\cdot), F(\cdot))$ we find

$$
\lim _{u \rightarrow-\infty} P\left[X \leqslant \frac{u}{x_{1}}, Y \leqslant \frac{u}{y_{1}} \mid X \leqslant \frac{u}{x_{2}}, Y \leqslant \frac{u}{y_{2}}\right]=\left(\frac{x_{1}^{-\alpha \beta}+y_{1}^{-\alpha \beta}}{x_{2}^{-\alpha \beta}+y_{2}^{-\alpha \beta}}\right)^{-1 / \alpha} .
$$


In this case we have a parametric family where we have to estimate only two parameters from the data.

Example 3.3 (Gumbel copula). Choose $x>0$. Set $u=-\log v \rightarrow \infty$ as $v \rightarrow 0$.

$$
\begin{aligned}
\lim _{v \rightarrow 0} \frac{C^{\mathrm{Gu}, \alpha}(x v, v)}{C^{\mathrm{Gu}, \alpha}(v, v)} & =\lim _{v \rightarrow 0} \frac{\exp \left[-\left\{(-\log x v)^{\alpha}+(-\log v)^{\alpha}\right\}^{1 / \alpha}\right]}{\exp \left[-\left\{2(-\log v)^{\alpha}\right\}^{1 / \alpha}\right]} \\
& =\lim _{u \rightarrow \infty} \exp \left[-\left\{(u-\log x)^{\alpha}+u^{\alpha}\right\}^{1 / \alpha}+2^{1 / \alpha} u\right] \\
& =\exp \left[2^{1 / \alpha-1} \log x\right]=x^{2^{1 / \alpha-1}}=g(x) .
\end{aligned}
$$

Hence

$$
G(x, y)=x^{2^{1 / \alpha-1}} \cdot y^{2^{1 / \alpha-1}}
$$

which means that asymptotically the random variables are independent (see also [8], Theorem 3.5). From an extreme value point of view this means, that the Gumbel copula generates excess values which are asymptotically independent. In practice this model may be problematic if one tries to analyze how dangereous joint extreme values are, since usually one underestimates the role of the dependence structure.

Remark. For Archimedean copulas the limit (2.6) is determined by the index of regular variation at zero of $\psi$ (see Theorem 3.3 in [8]).

\subsection{Bivariate Gaussian distribution}

We assume that $(X, Y)$ has a bivariate Gaussian distribution with standard normal marginals and correlation $\rho$, $|\rho|<1$. We denote the marginals by $\Phi$ and its density by $\varphi$. It follows from Sklar's Theorem that the Gaussian copula is given by

$$
C(x, y)=P\left[X \leqslant \Phi^{-1}(x), Y \leqslant \Phi^{-1}(y)\right] .
$$

Unfortunately there is no closed form for the Gaussian copula. Nevertheless we are able to calculate the function $g(\cdot)$ given in (2.6).

$$
g(x)=\lim _{v \rightarrow 0} \frac{C(x v, v)}{C(v, v)}=\lim _{u \rightarrow-\infty} \frac{C(x \Phi(u), \Phi(u))}{C(\Phi(u), \Phi(u))}=\lim _{u \rightarrow-\infty} \frac{P\left[X \leqslant \Phi^{-1}(x \Phi(u)), Y \leqslant u\right]}{P[X \leqslant u, Y \leqslant u]} .
$$

Hence we need to analyze the asymptotic behaviour of $x \Phi(u)$. From Mill's ratio we obtain (see [2], (3.38))

$$
\Phi(u) \sim \frac{1}{\sqrt{2 \pi}|u|} \exp \left\{-\frac{u^{2}}{2}\right\}, \quad \text { as } u \rightarrow-\infty .
$$

Hence we see that

$$
x \Phi(u) \sim \Phi\left(-\left(u^{2}-2 \log x\right)^{1 / 2}\right), \quad \text { as } u \rightarrow-\infty .
$$

This implies using l'Hôpital's rule

$$
\begin{aligned}
g(x)= & \lim _{u \rightarrow-\infty} \frac{P\left[X \leqslant-\left(u^{2}-2 \log x\right)^{1 / 2}, Y \leqslant u\right]}{P[X \leqslant u, Y \leqslant u]} \\
= & \lim _{u \rightarrow-\infty} \frac{P\left[X \leqslant-\left(u^{2}-2 \log x\right)^{1 / 2} \mid Y=u\right] \varphi(u)}{2 P[X \leqslant u \mid Y=u] \varphi(u)} \\
& -\frac{P\left[Y \leqslant u \mid X=-\left(u^{2}-2 \log x\right)^{1 / 2}\right] \varphi\left(-\left(u^{2}-2 \log x\right)^{1 / 2}\right)\left(u^{2}-2 \log x\right)^{-1 / 2} u}{2 P[X \leqslant u \mid Y=u] \varphi(u)} .
\end{aligned}
$$


Using a similar estimate for the density $\varphi$ as in (3.13), (3.14) we see that the last term simplifies so that we can write

$$
\begin{aligned}
g(x) & =\lim _{u \rightarrow-\infty} \frac{P\left[X \leqslant-\left(u^{2}-2 \log x\right)^{1 / 2} \mid Y=u\right]-x P\left[Y \leqslant u \mid X=-\left(u^{2}-2 \log x\right)^{1 / 2}\right]}{2 P[X \leqslant u \mid Y=u]} \\
& =\lim _{u \rightarrow-\infty} \frac{\Phi\left(-\frac{\left(u^{2}-2 \log x\right)^{1 / 2}+\rho u}{\left(1-\rho^{2}\right)^{1 / 2}}\right)-x \Phi\left(\frac{u+\rho\left(u^{2}-2 \log x\right)^{1 / 2}}{\left(1-\rho^{2}\right)^{1 / 2}}\right)}{2 \Phi\left(\frac{u-\rho u}{\left(1-\rho^{2}\right)^{1 / 2}}\right)},
\end{aligned}
$$

where in the last step we have used that the conditional distribution of $X \mid\{Y=y\}$ is again normally distributed with mean $\rho y$ and variance $1-\rho^{2}$. Using l'Hôpital's rule once again one finds that (we skip this tedious calculation)

$$
g(x)=x^{1 /(1+\rho)} .
$$

Hence $\theta=-2 /(1+\rho)$ and $G(x, y)=x^{1 /(1+\rho)} \cdot y^{1 /(1+\rho)}$, which means that asymptotically bivariate Gaussian random variables are independent. Furthermore, the marginals $\Phi$ belong to the Gumbel case with $a(u)=-1 / u$ (see e.g. [2], Example 3.3.29). Hence using Theorem 2.5

$$
\begin{aligned}
& \lim _{u \rightarrow-\infty} P\left[X \leqslant u-\frac{x_{1}}{u}, Y \leqslant u-\frac{y_{1}}{u} \mid X \leqslant u-\frac{x_{2}}{u}, Y \leqslant u-\frac{y_{2}}{u}\right] \\
& \quad=\exp \left\{\frac{1}{1+\rho}\left[x_{1}-x_{2}\right]\right\} \cdot \exp \left\{\frac{1}{1+\rho}\left[y_{1}-y_{2}\right]\right\} .
\end{aligned}
$$

Conclusion. The Gaussian distribution defines a model which has asymptotically independent excess distributions (the joint excess dependence structure converges to independence). In finance one often logarithmizes the data and then one assumes multivariate normality: also this log-normal model is asymptotically independent, since logarithmizing the data has only an effect on the marginal distributions but not on the dependence structure (see Sklar's theorem (Theorem 2.2) which says that the copula is invariante under monotonically increasing transformations). I.e. also in this logarithmized world we have asymptotically independent excess distributions.

\section{Proofs}

Proof of Lemma 2.4. Since $C$ is a distribution on the unit cube with uniform marginals it is clear that $g$ is increasing with $g(0)=0$ and $g(1)=1$. Consider $x, y>0$ then we have, using the symmetry of $(X, Y)$

$$
\begin{aligned}
\lim _{u \rightarrow 0} \frac{C(x u, y u)}{C(u, u)} & =\lim _{u \rightarrow 0} \frac{C(x u, y / x \cdot x u)}{C(x u, x u)} \frac{C(x u, x u)}{C(x u, 1 / x \cdot x u)} \frac{C(x u, u)}{C(u, u)} \\
& =g\left(\frac{y}{x}\right) \cdot 1 / g\left(\frac{1}{x}\right) \cdot g(x) \\
& =g\left(\frac{x}{y}\right) \cdot 1 / g\left(\frac{1}{y}\right) \cdot g(y),
\end{aligned}
$$

where in the last step we have used that the problem is completely symmetric in $x$ and $y$.

For $z>0$ we define $(g(z)>0$ for $z>0)$

$$
f(z)=\frac{g(z)}{g(1 / z)} .
$$

Hence $f$ satisfies $f(1)=1$ and (4.1) implies

$$
f(y / x)=\frac{f(y)}{f(x)} .
$$


But this implies, if we set $y=1$, that $f(1 / x)=1 / f(x)$. Hence (4.3) can be rewriten as (set $z=1 / x)$

$$
f(y z)=f(y / x)=f(y) \cdot f(1 / x)=f(y) \cdot f(z) .
$$

Formula (4.4) is known as Hamel's functional equation whose unique solution is $f(x)=x^{\theta}$, for some $\theta \in \mathbb{R}$ (see, e.g., [14] Theorem 1.4). Hence from (4.2) we have $z^{\theta} \cdot g(1 / z)=g(z)$ for $z>0$. This finishes the proof of Lemma 2.4 .

Proof of Theorem 2.5. Since we assume that the limit in (2.6) exists, we implicitely assume that $C(u, u)>0$ for all $u>0$ ( $C$ is increasing in both arguments). Hence the conditional probabilities in the statements of Theorem 2.5 are well-defined.

(a) Fréchet case: Assume $F(\cdot) \in \mathcal{R}_{-\beta}, \beta>0$. Hence for all $x, \varepsilon>0$ there exists $u_{0}$ such that for all $u<u_{0}$

$$
(x-\varepsilon)^{\beta} F(u) \leqslant F(u / x) \leqslant(x+\varepsilon)^{\beta} F(u) .
$$

This implies for all $\varepsilon>0,0<x_{1} \leqslant x_{2}, 0<y_{1} \leqslant y_{2}$ and $u<0$ sufficiently small

$$
\begin{aligned}
P\left[X \leqslant \frac{u}{x_{1}}, Y \leqslant \frac{u}{y_{1}} \mid X \leqslant \frac{u}{x_{2}}, Y \leqslant \frac{u}{y_{2}}\right] & =\frac{P\left[X \leqslant u / x_{1}, Y \leqslant u / y_{1}\right]}{P\left[X \leqslant u / x_{2}, Y \leqslant u / y_{2}\right]} \\
& =\frac{C\left(F\left(u / x_{1}\right), F\left(u / y_{1}\right)\right)}{C\left(F\left(u / x_{2}\right), F\left(u / y_{2}\right)\right)} \\
& \leqslant \frac{C\left(\left(x_{1}+\varepsilon\right)^{\beta} F(u),\left(y_{1}+\varepsilon\right)^{\beta} F(u)\right)}{C\left(\left(x_{2}-\varepsilon\right)^{\beta} F(u),\left(y_{2}-\varepsilon\right)^{\beta} F(u)\right)} .
\end{aligned}
$$

We set $v=F(u) \rightarrow 0$ (as $u \rightarrow-\infty)$. Using a similar decomposition as in Lemma 2.4 we obtain

$$
\begin{aligned}
& \limsup _{u \rightarrow-\infty} P\left[X \leqslant \frac{u}{x_{1}}, Y \leqslant \frac{u}{y_{1}} \mid X \leqslant \frac{u}{x_{2}}, Y \leqslant \frac{u}{y_{2}}\right] \leqslant \limsup _{v \rightarrow 0} \frac{C\left(\left(x_{1}+\varepsilon\right)^{\beta} v,\left(y_{1}+\varepsilon\right)^{\beta} v\right)}{C\left(\left(x_{2}-\varepsilon\right)^{\beta} v,\left(y_{2}-\varepsilon\right)^{\beta} v\right)} \\
& \quad=\frac{g\left(\left(\left(y_{1}+\varepsilon\right) /\left(x_{1}+\varepsilon\right)\right)^{\beta}\right) \cdot 1 / g\left(\left(1 /\left(x_{1}+\varepsilon\right)\right)^{\beta}\right) \cdot g\left(\left(x_{1}+\varepsilon\right)^{\beta}\right)}{g\left(\left(\left(y_{2}-\varepsilon\right) /\left(x_{2}-\varepsilon\right)\right)^{\beta}\right) \cdot 1 / g\left(\left(1 /\left(x_{2}-\varepsilon\right)\right)^{\beta}\right) \cdot g\left(\left(x_{2}-\varepsilon\right)^{\beta}\right)} \\
& =\frac{\left(x_{1}+\varepsilon\right)^{\theta \beta} \cdot g\left(\left(\left(y_{1}+\varepsilon\right) /\left(x_{1}+\varepsilon\right)\right)^{\beta}\right)}{\left(x_{2}-\varepsilon\right)^{\theta \beta} \cdot g\left(\left(\left(y_{2}-\varepsilon\right) /\left(x_{2}-\varepsilon\right)\right)^{\beta}\right)},
\end{aligned}
$$

where $\theta$ is given by Lemma 2.4. Of course we can find a similar lower bound for the lim inf. Now since $\varepsilon>0$ can be chosen arbitrarily small we find (using the continuity of the function $g$ )

$$
\lim _{u \rightarrow-\infty} P\left[X \leqslant \frac{u}{x_{1}}, Y \leqslant \frac{u}{y_{1}} \mid X \leqslant \frac{u}{x_{2}}, Y \leqslant \frac{u}{y_{2}}\right]=\frac{x_{1}^{\theta \beta} \cdot g\left(\left(y_{1} / x_{1}\right)^{\beta}\right)}{x_{2}^{\theta \beta} \cdot g\left(\left(y_{2} / x_{2}\right)^{\beta}\right)}=\frac{G\left(x_{1}^{\beta}, y_{1}^{\beta}\right)}{G\left(x_{2}^{\beta}, y_{2}^{\beta}\right)} .
$$

This finishes the proof in the Fréchet case.

(b) Weibull case: Assume $F$ satisfies $x_{F}>-\infty$ and $F\left(x_{F}-1 / \cdot\right) \in \mathcal{R}_{-\beta}, \beta>0$. Hence for all $x, \varepsilon>0$ there exists $u_{0}<0$ such that for all $u<u_{0}$

$$
(x-\varepsilon)^{\beta} F\left(x_{F}-1 / u\right) \leqslant F\left(x_{F}-x / u\right) \leqslant(x+\varepsilon)^{\beta} F\left(x_{F}-1 / u\right) .
$$

But then the claim follows as in the Fréchet case if we set $v=F\left(x_{F}-1 / u\right) \rightarrow 0$ as $u \rightarrow-\infty$. This finishes the proof in the Weibull case.

(c) Gumbel case: Assume $F$ satisfies $x_{F} \geqslant-\infty$ and there exists a positive function $a(\cdot)$ such that (2.4) is satisfied. Hence for all $x, \varepsilon>0$ there exists $u_{0}>x_{F}$ such that for all $x_{F}<u<u_{0}$

$$
\mathrm{e}^{x-\varepsilon} F(u) \leqslant F(u+x a(u)) \leqslant \mathrm{e}^{x+\varepsilon} F(u) .
$$


But then the claim follows as in the Fréchet case if we replace $x^{\beta}$ by $\mathrm{e}^{x}$ and if we set $v=F(u) \rightarrow 0$ as $u \rightarrow x_{F}$. This finishes the proof in the Gumbel case.

This finishes the proof of Theorem 2.5.

Proof of Lemma 2.6. The positivity of $G$ follows from the positivity of $g$. We first prove that $G(\cdot, \cdot)$ is symmetric: If one coordinate is 0 the statement is clear. Hence choose $x, y>0$. Using Lemma 2.4

$$
G(x, y)=y^{\theta} g\left(\frac{x}{y}\right)=y^{\theta}\left(\frac{x}{y}\right)^{\theta} g\left(\frac{y}{x}\right)=x^{\theta} g\left(\frac{y}{x}\right)=G(y, x) .
$$

Using the symmetry it suffices to prove the continuity and the increasing property for the first argument. The continuity easily follows from the continuity of $g$. For the increasing property we need to prove that $g$ is increasing, but this is immediately clear since $C(u x, u)$ is a distribution, hence increasing in $x$.

Finally, there remains to prove the two-increasing property: For $0 \leqslant z_{1} \leqslant z_{2}, 0 \leqslant w_{1} \leqslant w_{2}$ the condition

$$
G\left(z_{2}, w_{2}\right)-G\left(z_{2}, w_{1}\right)-G\left(z_{1}, w_{2}\right)+G\left(z_{1}, w_{1}\right) \geqslant 0
$$

needs to be satisfied. This ensures that we obtain (after suitable normalization) a two dimensional probability distribution. It essentially means that a random vector $\left(Z_{1}, Z_{2}\right) \stackrel{(d)}{\sim} G(\cdot, \cdot) / G\left(x_{2}, y_{2}\right)$ assigns positive weights to the probabilities $P\left[z_{1} \leqslant Z_{1} \leqslant z_{2}, w_{1} \leqslant Z_{2} \leqslant w_{2}\right]$.

Choose $F(\cdot) \in \mathcal{R}_{-1}$ hence we are in the Fréchet case (in the examples section we have seen the existence of such marginal distributions). Hence we define for $0<x_{1} \leqslant x_{2}$ and $0<y_{1} \leqslant y_{2}$

$$
P_{u}\left(x_{1}, y_{1} ; x_{2}, y_{2}\right)=P\left[X \leqslant \frac{u}{x_{1}}, Y \leqslant \frac{u}{y_{1}} \mid X \leqslant \frac{u}{x_{2}}, Y \leqslant \frac{u}{y_{2}}\right] .
$$

Since $P_{u}\left(\cdot, \cdot ; x_{2}, y_{2}\right)$ is a distribution on $\left[0, x_{2}\right] \times\left[0, y_{2}\right]$ for all $u<0$, it satisfies the two-inceasing property for all $u<0$. But this implies that also the limit (as $u \rightarrow-\infty) G(\cdot, \cdot) / G\left(x_{2}, y_{2}\right)$ satisfies the two-increasing property. This finishes the proof of the lemma.

\section{References}

[1] A.A. Balkema, L. de Haan, Residual lifetime at great age, Ann. Probab. 2 (1974) 792-804.

[2] P. Embrechts, C. Klüppelberg, T. Mikosch, Modelling Extremal Events for Insurance and Finance, Springer, Berlin, 1997.

[3] P. Embrechts, A. McNeil, D. Straumann, Correlation and dependency in risk management: properties and pitfalls, in: M. Dempster (Ed.), Risk Management: Value at Risk and Beyond, Cambridge University Press, Cambridge, 2002, pp. 176-223.

[4] W.E. Frees, E.A. Valdez, Understanding relationships using copulas, North Amer. Actuarial J. 2 (1998) 1-25.

[5] C. Genest, J. MacKay, Copules archimediennes et familles de lois bidimensionelles dont les marges sont donnees, Canadian J. Statist. 14 (1986) $154-159$

[6] C. Genest, J. MacKay, The joy of copulas: Bivariate distributions with uniform marginals, The American Statistican 40 (1986) $280-283$.

[7] H. Joe, Multivariate Models and Dependence Concepts, Chapman \& Hall, London, 1997.

[8] A. Juri, M.V. Wüthrich, Copula convergence theorems for tail events, Insurance: Math. Econom. 30 (3) (2002) $405-420$.

[9] A. Juri, M.V. Wüthrich, Tail dependence from a distributional point of view, Preprint, 2003.

[10] S. Kotz, S. Nadarajah, Extreme Value Distributions, Imperial College Press, London, 2000.

[11] R.B. Nelsen, An Introduction to Copulas, Springer, New York, 1999.

[12] J. Pickands III, Statistical inference using extreme order statistics, Ann. Statist. 3 (1975) 119-131.

[13] B. Schweizer, A. Sklar, Probabilistic Metric Spaces, North-Holland, New York, 1983.

[14] E. Senata, Regularly Varying Functions, in: Lecture Notes in Math., Springer, Heidelberg, 1976.

[15] A. Sklar, Fonctions de répartition à $n$ dimensions et leurs marges, Publications de l'Institut de Statistique de l'Université de Paris 8 (1959) 229-231.

[16] M.V. Wüthrich, Asymptotic value-at-risk estimates for sums of dependent random variables, Astin Bull. 33 (1) (2003) 75-92. 